\title{
EFFERENT ACTIVITY IN RENAL AND INTESTINAL NERVES DURING CIRCULATORY REFLEXES*
}

\author{
Hiroshi IrISAwa, Ishio NinOmiya and Gray Woolley** \\ Department of Physiology, School of Medicine, Hiroshima University, \\ Kasumi-cho, Hiroshima
}

\begin{abstract}
Summary The effects of baroceptor reflex on the sympathetic nerve activity to the intestine (INA) and kidney (RNA) were compared in anesthetized cats. Grouped discharges synchronous with the cardiac cycle was not the major pattern in INA and approximately $73 \%$ of the total INA was composed of a continuous or fluctuating pattern. With increase in mean aortic pressure from 116 to $216 \mathrm{mmHg}$ after administration of norepinephrine, INA showed various response patterns: initial inhibition followed by an increase (Type A), increase without inhibition (Type B), slight inhibition (Type C) and no change (Type D), whereas RNA decreased to the noise level. Type A and B were the most common response patterns. In the open loop condition, initial inhibition in Type A disappeared, while the increase of INA in Types A and B remained unchanged. When RNA was completely inhibited by baroceptor inputs during occlusion of the descending thoracic aorta, INA was not inhibited but rather slightly increased. It is concluded that INA showed quantitatively and qualitatively different responses to baroceptor inputs as compared to RNA.
\end{abstract}

The resistance vessels in the various peripheral vascular beds do not respond uniformly to the baroceptor activity in the aortic arch and carotid sinus (HADJmINAS and ÖBerg, 1968; Heymans and NeIL, 1958). These differences in response may be due in part at least to non-uniform distribution of the sympathetic nerve activity (SNA) to different organs (GREEN and HeFrron, 1966; IrIKI et al., 1971; NinomiYa et al., 1971, 1973; Nisimaru, 1971; Weidinger and Fedina, 1960). This in turn may be due to richer or sparser innervation of resistance vessels in some regions or to more or less baroceptor-associated traffic in the sym-

Received for publication October 18, 1973

* This study was supported by a research grant from the Japanese Ministry of Education to H. Irisawa and I. Ninomiya

** Present address: Department of Physiology, Monash University, Clayton, Victoria, Australia 入沢 宏, 二宮石雄 
pathetic supply reaching the particular vessels.

The SNA to the spleen, kidney, heart, stomach, and skin show different responses to baroceptor reffexes. Thus most of the SNA to the spleen, kidney, and heart shows complete inhibition during the baroceptor depressor reflex (NinOMiYa et al., 1971), but about $80 \%$ of the SNA to the stomach is independent of baroceptor function (NISIMARU, 1971). Most of the SNA to the skin is also baroceptor independent (NiNOMIYA et al., 1973).

Although there has been ample evidence for baroceptor reflexes affecting intestinal blood flow (GRAYSON and MENDEL, 1965), the intestinal vascular bed seems to be only moderately involved in baroceptor reflex as compared with the muscle resistance vessels (CElANDER and Folkow, 1951; Folkow, 1967; ÖBerG, 1964). These results suggest that the baroceptor reflexes may have small effects on the SNA to intestinal resistance vessels as compared with other vascular beds. However, little relevant information is available concerning intestinal nerve activity. The present report describes the recording of postganglionic sympathetic nerve activity to the intestine as compared with renal nerve activity in conjunction with various baroceptor inputs.

\section{METHODS}

Eleven cats $(2-3 \mathrm{~kg})$ were used in this study. After the animal was anaesthetized with sodium pentobarbital (Nembutal $35 \mathrm{mg} / \mathrm{kg}$, ip) the trachea was intubated. On those occasions when the thorax was opened for aortic occlusion, the lungs were ventilated by intermittent positive pressure from a Harvard respiratory pump (Model 606).

Additional doses of sodium pentobarbital $(5 \mathrm{mg} / \mathrm{kg}$, iv) were injected intravenously through a catheter inserted in the left femoral vein whenever responses to pain were detected.

The intestinal nerve was separated from the sheath surrounding a branch of the mesenteric vessels near the intestinal wall, i.e. ileum or jejunum. The method of identifying the renal nerve was the same as previously described (NINOMIYA et al., 1971). The efferent nerve discharges were recorded central to the cut end of the nerve bundle. The recording of the nerve impulses and the integration of the original neurogram have been described previously (NinOMIYA et al., 1971). The original neurogram was continuously monitored by a cathode ray oscilloscope (Tektronix 502 A). The time averaging of the aortic pressure and intestinal and renal nerve activities was performed simultaneously by a hybrid computer (Hidas 200, Hitachi Ltd.). The output of the computer was displayed on an $\mathrm{XY}$ recorder.

\section{RESULTS}

1. Comparison of the intestinal nerve activity and the renal nerve activity 
Generally, grouped discharges synchronous with but of delayed phase with respect to the cardiac pressure cycle and its respiratory modulation were readily observed in the spontaneous activity of the renal sympathetic nerve. However, continuous and fluctuating discharges appeared dominant in the intestinal nerve (Fig. 1).


Fig. 1. An example of the original neurogram. RNA, Renal nerve activity; INA, intestinal nerve activity; AP, aortic pressure.

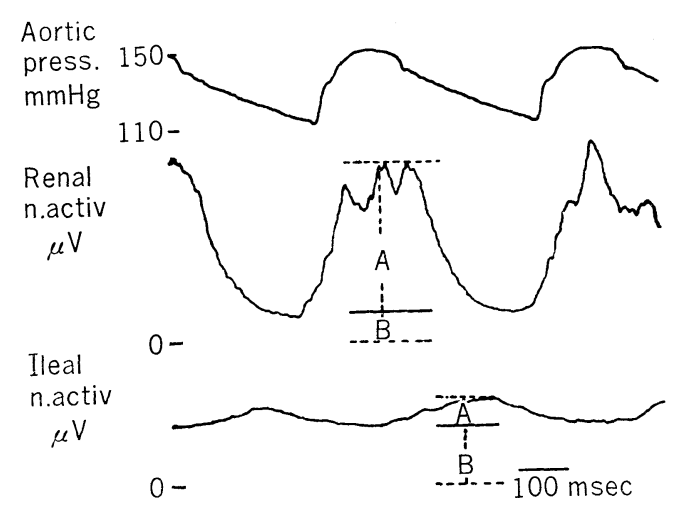

Fig. 2. Comparison of the grouping (A) and tonic (B) nerve activities to the kidney and intestine obtained by averaging technique.

In order to detect any grouping of discharges synchronous with the cardiac cycle the integrated nerve activity was averaged over 20 to 50 cardiac cycles in 10 cats. An example of the averaged sympathetic nerve activity is shown in Fig. 2. A grouping (A in Fig. 2) and a tonic component (B in Fig. 2) were found in both 
the averaged RNA and INA. The absolute magnitude of the grouped nerve activity was larger in RNA than in INA, whereas that of the tonic nerve activity was larger in INA than in RNA. The absolute magnitude of SNA $(\mu \mathrm{V})$ varied between different animals. Therefore, the relative magnitude of grouping in SNA $(\mathrm{A} /(\mathrm{A}+\mathrm{B}) \times 100)$ for a given cardiac cycle was computed in each preparation for statistical analysis. The relative magnitude of grouping obtained from 47 trials was $27 \%$ in INA and $85 \%$ in RNA (Table 1A). These data in-

Table 1A. Grouping of sympathetic nerve activity to the kidney (RNA) and intestine (INA).

\begin{tabular}{cccc}
\hline & Trial No. & \multicolumn{2}{c}{ Grouping $(\%)$} \\
\cline { 2 - 4 } Cat No. & & RNA & INA \\
\hline 1 & 2 & 97.9 & 26.0 \\
2 & 7 & 89.5 & 38.0 \\
3 & 7 & 91.0 & 14.0 \\
4 & 1 & 81.8 & 41.5 \\
5 & 7 & 81.0 & 20.0 \\
6 & 10 & 66.6 & 28.1 \\
7 & 2 & 94.9 & 19.2 \\
8 & 5 & 86.9 & 32.1 \\
9 & 2 & 89.5 & 36.3 \\
10 & 47 & 77.0 & 17.4 \\
\hline 10 & & $85.6 * \pm 2.7 * *$ & $27.2 \pm 2.8$ \\
\hline
\end{tabular}

* Mean

** SE

Table 1B. Delay times of baroceptor reflex on sympathetic nerve activity to the kidney (RNA) and intestine (INA).

\begin{tabular}{rrrrrc}
$\begin{array}{r}\text { Cat } \\
\text { No. }\end{array}$ & $\begin{array}{c}\text { Trial } \\
\text { No. }\end{array}$ & $\begin{array}{c}\text { Interval of } \\
\text { cardiac cycle }\end{array}$ & \multicolumn{2}{c}{ Delay time, msec } & Difference in \\
\cline { 5 - 6 } & RNA & INA & delay time, msec \\
\hline 1 & 4 & 385 & 185 & 372 & 187 \\
2 & 1 & 350 & 190 & 330 & 140 \\
3 & 3 & 496 & 163 & 363 & 200 \\
4 & 7 & 434 & 170 & 324 & 154 \\
5 & 4 & 527 & 197 & 332 & 135 \\
6 & 2 & 485 & 185 & 345 & 160 \\
7 & 2 & 510 & 210 & 360 & 150 \\
8 & 4 & 325 & 175 & 262 & 87 \\
9 & 2 & 270 & 195 & 328 & 133 \\
10 & 2 & 375 & 190 & 355 & 127 \\
11 & 3 & 270 & 180 & 307 & $148.9 \pm 10.1$ \\
\hline 11 & 34 & $402.4^{*} \pm 27.0^{* *}$ & $185.4 \pm 3.8$ & $334.3 \pm 10.3$ & \\
\hline
\end{tabular}

* Mean

** SE 
dicate that RNA consists predominantly of the grouped component while INA consists of mainly the tonic component.

Assuming that the peak INA coincides with the onset of sympathetic inhibition as in the case of RNA, the time interval between the maximum positive $d p / d t$ of aortic pressure and peak SNA in the same cardiac cycle was measured in 11 cats. The interval was $334 \pm 10 \mathrm{msec}$ in INA and $185 \pm 4 \mathrm{msec}$ in RNA. INA lagged $149 \mathrm{msec}$ behind RNA (Table 1B).

\section{Baroceptor reflex effect on SNA to the intestine and kidney}

a) Effect of administration of norepinephrine on SNA with 4 major afferent pathways intact. After the intravenous administration of $10 \mu \mathrm{g} / \mathrm{kg}$ of norepinephrine, complete inhibition of RNA was observed in all 35 trials of 10 cats. On the other hand, the time course of INA in response to norepinephrine administration varied from animal to animal and from experiment to experiment. Responses were classified into four patterns (Table 2). When mean aortic pressure (MAP) increased by $86 \pm 17 \%$ of the control value $(116 \pm 8 \mathrm{mmHg})$, INA measured in 18 trials was inhibited initially to $67 \%$ of the control level which was followed by $7 \%$ increase above the control value (Fig. 3A and Type A in Table 2). In 11 other trials, INA showed only an increase of $116 \%$ above the control value when MAP increased $61 \pm 6 \%$ above the control pressure of $131 \pm 5 \mathrm{mmHg}$ (Fig. 3B and Type B in Table 2). INA showed a slight inhibition in 2 trials (Type $\mathrm{C}$ in Table 2) and no detectable change in 4 trials (Type $\mathrm{D}$ in Table 2).

Table 2. Frequency distribution of patterns to administration of norepinephrine.

\begin{tabular}{cccccc}
\hline Cat No. & Trial No. & \multicolumn{5}{c}{$\begin{array}{c}\text { Response patterns (type) of mean } \\
\text { intestinal nerve activity }\end{array}$} \\
\hline 1 & & A & B & C & D \\
2 & 5 & 4 & 1 & 0 & 0 \\
3 & 3 & 2 & 1 & 0 & 0 \\
4 & 5 & 0 & 3 & 1 & 1 \\
5 & 9 & 7 & 0 & 1 & 0 \\
6 & 4 & 0 & 2 & 0 & 2 \\
7 & 3 & 2 & 1 & 0 & 0 \\
8 & 2 & 1 & 1 & 0 & 0 \\
9 & 3 & 1 & 1 & 0 & 0 \\
10 & 2 & 1 & 0 & 0 & 0 \\
10 & 2 & 0 & 1 & 0 & 1 \\
\hline
\end{tabular}

Type A, decrease and then increase; Type B, increase; Type C, decrease; Type D, no change.

An example of the averaged pattern synchronous with cardiac cycle obtained before and after administration of norepinephrine is shown in Fig. 4. After the administration of norepinephrine, grouped components in both INA and RNA 

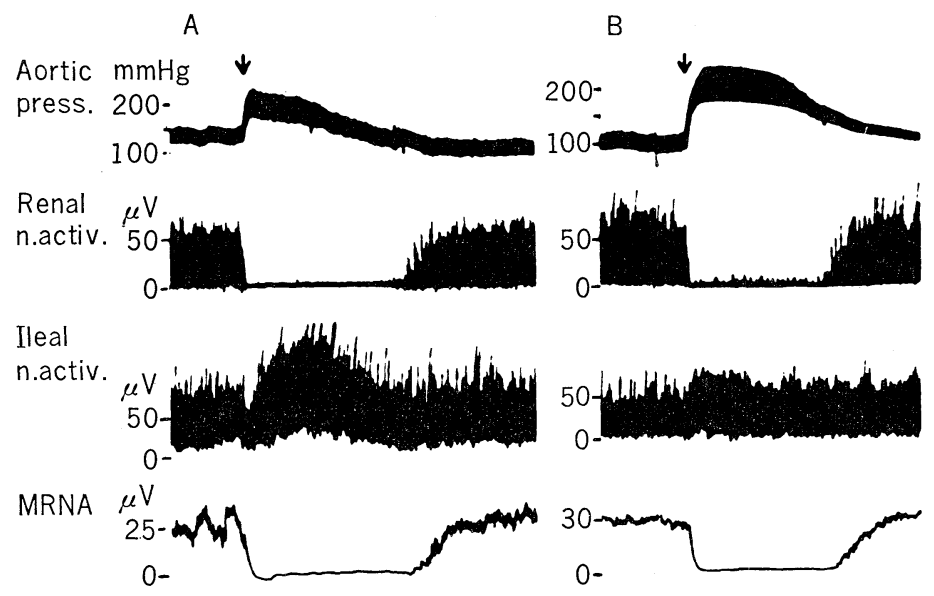

MINA
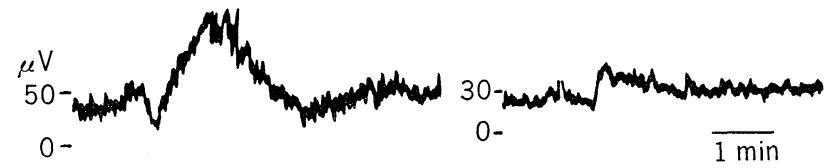

Fig. 3. Norepinephrine $(10 \mu \mathrm{g} / \mathrm{kg}$ iv) was administered at arrows. Two types of responses (Types A and B) in INA are shown in A and B, respectively. Renal nerve activity completely abolished as aortic pressure rose.

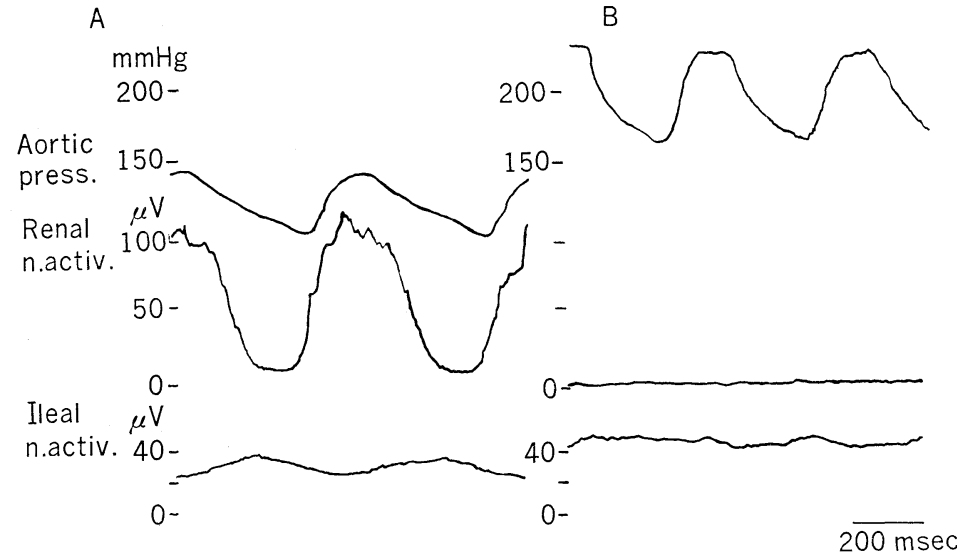

Fig. 4. Comparison of the averaged nerve activities to the kidney and intestine at the control (A) and after the administration of norepinephrine (B).

and the tonic component in RNA disappeared, but the tonic component in INA increased against the increase in baroceptor inputs (Fig. 4).

b) Effect of administration of norepinephrine on SNA with opened afferent loops. Before the open loop experiment, administration of norepinephrine caused complete inhibition in RNA and slight inhibition which was followed by a signi- 
ficant increase in INA. After bilateral vagotomy, both common carotid arteries were occluded to provide an open loop condition.

Under this circumstance, SNA to kidney was not inhibited by the baroceptor input due to the administration of norepinephrine. On the other hand, the increase in INA following the administration of norepinephrine was still observed. Similar phenomenon was recorded in 3 other experiments. These results indicate that the increase in INA caused by the administration of norepinephrine is independent of the baroceptor input.

In the controls, with the four major afferent pathways intact, the averaged SNA to the kidney and intestine show typically a grouped pattern synchronous with cardiac cycle (Fig. 5, thin lines). When norepinephrine was administered under open loop conditions, the grouped pattern in INA as well as in RNA disappeared, although both pulse and mean aortic pressures increased (Fig. 5, thick lines). Tonic SNA to the intestine increased above the initial control level.
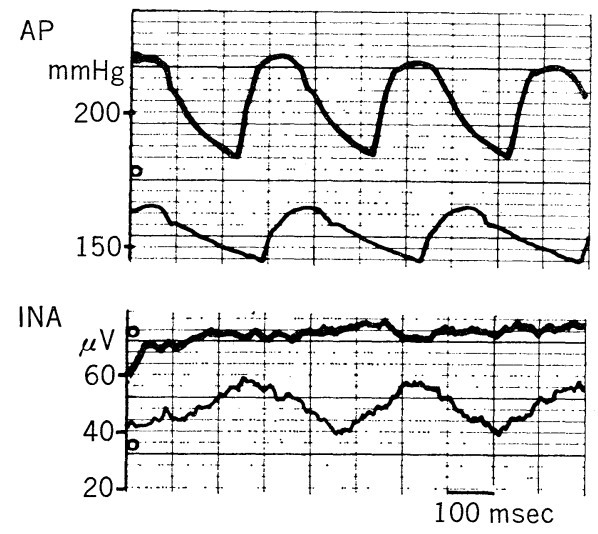

Fig. 5. Effect of opening 4 major baroceptor inputs on aortic pressure (AP) and intestinal nerve activity (INA). Averaged aortic pressure and nerve activities are obtained at control (thin line) and after the blocking of carotid sinus nerves by local application of $4 \%$ xyrocaine under bilateral vagotomy (thick line). The grouping activity of INA disappeared, but the tonic activity increased.

c) Effects of occlusion of the descending aorta on SNA. In 3 cats, sympathetic nerve activity to the kidney and intestine were measured simultaneously before and during occlusion of the descending thoracic aorta. Data are summarized in Table 3. When MAP increased above the control value, in 2 or 3 cats INA showed very little change (Fig. 6A), but in one cat INA showed an increase after slight initial inhibition (Fig. 6B). On the other hand, RNA was inhibited almost completely during occlusion. During occlusion of the descending thoracic aorta the grouped discharges synchronous with the cardiac cycle were abolished both in INA and RNA. 
Table 3. Effect of occlusion of descending thoracic aorta on mean aortic pressure (MAP), renal nerve activity (RNA), and intestinal nerve activity (INA).

\begin{tabular}{cccccc}
\hline & & \multicolumn{2}{c}{ MAP } & \multicolumn{2}{c}{ Occlusion (\% change) } \\
\cline { 3 - 6 } Cat No. & Trial No. & $\begin{array}{c}\text { Control value } \\
(\mathrm{mm} \mathrm{Hg})\end{array}$ & $\begin{array}{c}\text { Increase } \\
(\%)\end{array}$ & RNA & INA \\
\hline 1 & 6 & $158^{*} \pm 4.4^{* *}$ & $36.7 \pm 3$ & $-93.9 \pm 2$ & $+1.6 \pm 0.7$ \\
2 & 3 & $103 \pm 3.0$ & $46.7 \pm 5$ & $-91.0 \pm 7$ & $+5.9 \pm 1.6$ \\
3 & 12 & $130 \pm 8.4$ & $41.7 \pm 3$ & $-97.7 \pm 2$ & $+51.4 \pm 14.3$ \\
\hline
\end{tabular}

* Mean

** SE

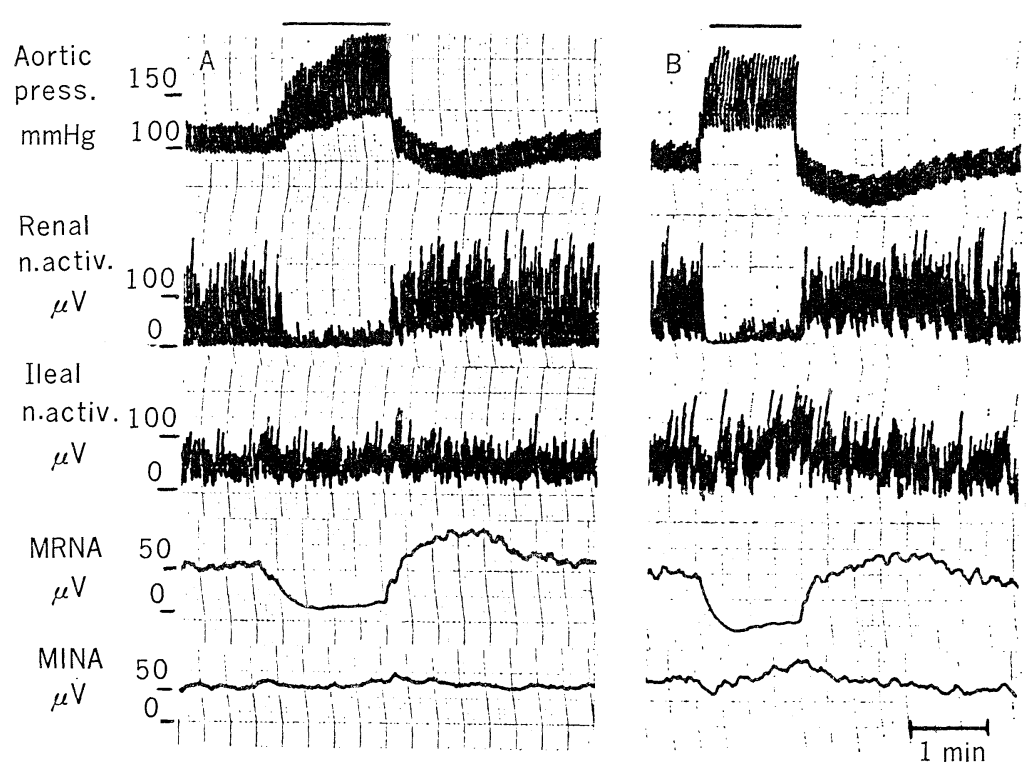

Fig. 6. Effect of occlusion of the descending aorta (bar) on the aortic pressure, renal nerve activity and ileal nerve activity. Mean renal nerve activity (MRNA) and mean intestinal nerve activity (MINA) are also shown. Two types of responses (A and B) were observed.

\section{DISCUSSION}

Nonuniform distribution of SNA to different parts of the body has been studied by several investigators (IRIKI et al., 1971; NINOMIYA et al., 1971, 1973; Nisimaru, 1971). Within visceral sympathetic nerves, SNA to the stomach and the gut were different from RNA. Both gastric nerve and intestinal nerve carry baroceptor independent activity, which has been shown to be only a minor part of the activity of the cardiac, renal and splenic nerves. This is to be expected since a large number of post-ganglionic sympathetic fibers terminate on the non- 
vascular structures of the intestine, viz. the intestinal smooth muscle and the enteric plexus. There is, however, an identifiable component of INA which is associated with rate-proportional baroceptor activity.

Whereas about $20 \%$ of SNA to the stomach was dependent on baroceptor activity, in the present experiments $27 \%$ (range: 14 to $41 \%$ ) of the total INA depended on baroceptor activity. This suggests that the baroceptor-dependent component of SNA throughout the gastrointestinal tract is approximately of the same magnitude. This activity may reasonably be inferred to reach the vascular structures. However, the distribution of this activity to the various vascular components of the tract remains unknown. Furthermore, the final potency of this activity in conferring resistive or capacitive change to the vasculature is also unknown. Experiments in which direct electrical stimulation was applied to the nerve did not illuminate this, since the stimulation bears little relation in intensity and temporal or spacial distribution to the natural neural traffic. A further complication exists that some of SNA which is independent of baroceptor activity may nevertheless be reaching the vascular structures. The present experiments could not distinguish such traffic.

The grouped nerve activity in INA and the temporal inhibition of INA during norepinephrine injection are considered to be of baroceptor origin. Therefore, the time interval between the maximum positive $d p / d t$ of the aortic pressure and the peak of SNA in the same cardiac cycle was measured as an index of delay time of baroceptor to the different SNA. In SNA to the heart, kidney, spleen, stomach and intestine it was 164, 199, 292, 291, and $322 \mathrm{msec}$, respectively (NISIMARU, 1971). Skin nerve activity lagged behind RNA by $125 \mathrm{msec}$ (ÖBERG, 1964). It can therefore be stated that SNA to the skin and intestine show longer delay times than SNA to the kidney, heart and stomach. This difference in delay time is considered to be largely due to the postganglionic conduction time. Since our recording electrode was placed close to the intestinal wall, the length of conduction probably must be longest within the above-mentioned sympathetic nerves. Because of the variety of delay times in different SNA, the baroceptor effects are not synchronized at various sites.

Skin nerve activity has been shown to increase gradually against the baroceptor inputs during occlusion of the descending thoracic aorta (NINOMIYA et al., 1973). A similar phenomenon was observed in SNA to the intestine. The increase of INA during norepinephrine administration is in a direction opposite to the expected effect of the four major baroceptor reflex. It has been shown that the injection of epinephrine or norepinephrine causes the portal blood pressure to increase (SEMBA, 1954; SwAN and Reynolds, 1971). Although the origin of the increase in INA during norepinephrine administration is not yet clear, recent findings (ANDREWs et al., 1972) of the presence of mesentery "venous volume" or "stretch" receptors in the portal vein suggest that norepinephrine caused the portal blood pressure to increase and this in turn caused an increase 
in the efferent nerve discharge to the intestine. Thus it is possible that the increase in SNA to the intestine contrary to the baroceptor reflex after norepinephrine administration could be explained by the stimulation of these stretch receptors in the portal vein. Another possibility for the increase in INA after norepinephrine injection is the direct action of this drug on the sympathetic ganglia. This possibility has no experimental support, as yet.

In a subsequent report (manuscript in preparation), we will show that mechanically increased tension of the intestinal wall causes an increase in intestinal nerve activity. However, norepinephrine causes relaxation of the intestinal wall and not increased tension in the wall. The increase of INA after norepinephrine therefore seems not due to the activation of the stretch receptors in the intestinal wall.

\section{REFERENCES}

Andrews, C. J. H., Andrews, W. H. H., and Orbach, J. (1972) A sympathetic reflex elicited by distension of mesenteric venous bed. J. Physiol., 226: 119-131.

CELANDER, O. and Folkow, B. (1951) Are parasympathetic vasodilator fibers involved in depressor reflex elicited from the baroceptor region? Acta Physiol. Scand., 23: 64-77.

FolKow, B. (1967) Regional adjustments of intestinal blood flow. Gastroenterology, 52: 423432.

Grayson, J. and Mendel, D. (1965) Physiology of the Splanchnic Circulation. Edward Arnold Ltd., London.

Green, J. H. and Hefronon, P. F. (1966) Simultaneous recording of sympathetic activity in different regions. J. Physiol., 185: 48p-50p.

HADJIMINAS, J. and ÖBERG, B. (1968) Effects of carotid baroceptor reflex on venous tone in skeletal muscle and intestine of the cat. Acta Physiol Scand., 72: 518-532.

Heyman, C. and Neil, E. (1958) Reflexogenic Areas of the Cardiovascular System. Little, Brown and Co., Boston.

Iriki, M., Walther, O.E., PleschKa, K., and Simon, E. (1971) Regional cutaneous and visceral sympathetic nerve activity during asphyxia in the anesthetized rabbit. Pflügers Arch., 322: 167-182.

Ninomiya, I. and Irisawa, H. (1969) Summation of baroceptor reflex effects on sympathetic nerve activities. Am. J. Physiol., 216: 1330-1336.

NinomiYA, I., IrisawA, A., and NisimarU, N. (1973) Non uniformity of sympathetic nerve activity to the skin and kidney. Am. J. Physiol., 224: 256-264.

NinomiYa, I., Nisimaru, N., and Irisawa, H. (1971) Sympathetic nerve activity to the spleen, kidney and heart in response to baroceptor input. Am. J. Physiol., 221: 1346-1351.

Nisimaru, N. (1971) Comparison of gastric and renal nerve activity. Am. J. Physiol., 220: 1303-1308.

ÖBerg, B. (1964) Effects of cardiovascular reflexes on net capillary fluid transfer. Acta Physiol. Scand., 62: (Suppl) 229: 3-98.

SEmba, T. (1954) The effect of adrenaline on the portal venous pressure. Jap. Circ. J., 18: 33-37.

SwAn, K. G. and Reynolds, D. G. (1971) Adrenergic mechanisms in canine mesenteric circulation. Am. J. Physiol., 220: 1779-1785.

Weidinger, H. and Fedina, L. (1960) Versuche zur Tonisierung medüllare sympathetischer Zentren. Pflügers Arch., 272: 55-56. 